\title{
Regulation of hepatic fatty acid oxidation and ketogenesis
}

\section{By Victor A. Zammit, Hannah Research Institute, Ayr KA6 ${ }_{5} H L$, Scotland}

The regulation of fatty acid oxidation in the liver is a highly integrated, multisite process. Indeed, the main aim of this short review is to emphasize the multiplicity of sites at which regulation can potentially be exerted. In addition to integration within the pathway of fatty acid oxidation itself, there is also complex integration with several other biochemical processes in the liver, namely glycerolipid and fatty acid syntheses, gluconeogenesis and oxidation of acetyl-CoA through the tricarboxylic acid cycle. Most of the regulatory properties of the enzymically-catalyzed (and other) steps involved have been described for the liver of the rat and, consequently, most of what follows necessarily relates to that species. However, the few comparative studies that have been made have not only yielded important information on the diversity of the regulation of fatty acid metabolism in different types of mammals and other vertebrates but have also helped in the understanding of the basic biochemical mechanisms involved in the regulation of fatty acid oxidation and ketogenesis. In addition, the more recent developments in the field have been made through comparative studies of liver metabolism in unmated, pregnant, lactating and weaned rats, which serve to illustrate the potential of studies in which the behaviour of one biochemical system is compared in different and entirely physiological situations. The various stages of the reproductive cycle appear to be particularly useful in this regard.

A diagrammatic representation of the steps involved in fatty acid oxidation and ketogenesis in the liver is given in Fig. r. Uptake of long-chain fatty acids from the circulation into the liver cell is followed by formation of long-chain acyl-CoA esters (the metabolism of medium- and short-chain fatty acids will not be considered in this review). The partition of these esters between glycerolipid formation and acylcarnitine formation is the first branch-point which occurs in the pathway. Acylcarnitine esters are transported across the inner mitochondrial membrane and converted back to acyl-CoA esters which, through the process of $\beta$-oxidation, have successive two-carbon units removed to form acetyl-CoA. The disposal of acetyl$\mathrm{CoA}$ in mitochondria represents the second branch-point since this metabolite can be used either for the formation of acetoacetyl-CoA (and thence ketones) or citrate. The formation of acetoacetyl-CoA is the first step committed to the formation of acetoacetate via the 3-hydroxy-3-methylglutaryl-CoA pathway. Although the $\mathrm{NADH}$-linked formation of 3-hydroxybutyrate is not a universal property of the ketogenic process among vertebrate species (Zammit \& Newsholme, 1979; Zammit et al. 1979), it appears to be of regulatory importance in the liver of the rat.

The uptake of non-esterified fatty acids from the circulation by the liver is 


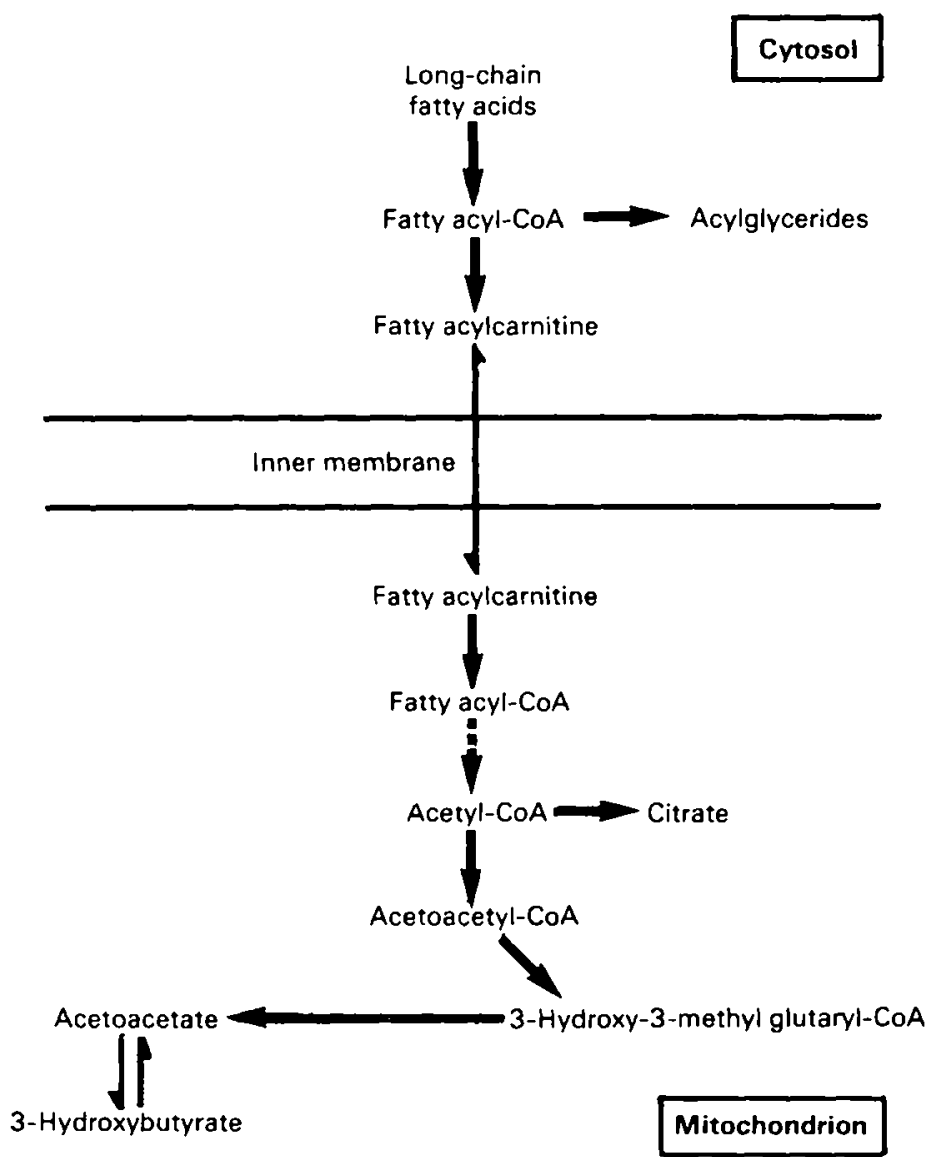

Fig. 1. Schematic outline of long-chain fatty acid metabolism and its compartmentation in the liver.

concentration-dependent and is not saturated even at very high concentrations of plasma fatty acids (Fine \& Williams, I 960; Nestel \& Steinberg, 1963; Goresky et al. 1978). This enables the liver to respond to changes in the rate of fatty acid release from adipose tissue and ensures increased hepatic uptake of fatty acids in physiological conditions which are characterized by increased lipolysis of triacylglycerols in adipose tissue. Indeed, it could be suggested that the regulation of hepatic fatty acid oxidation and ketogenesis occurs, in part, outside the liver, at the level of triacylglycerol lipase activity in adipose tissue (Owen et al. 1969; Hales et al. 1978; Williamson \& Whitelaw, 1978). Thus, in most vertebrate species the concentration of non-esterified fatty acids in the plasma increases in ketogenic states (Table 1), the only exception being the elasmobranch fishes (Zammit \& Newsholme, 1979) in which triacylglycerols are stored in the liver rather than in a specialized extrahepatic storage tissue.

The ATP-dependent formation of long-chain acyl-CoA esters occurs in the extramitochondrial compartment of the hepatocytes. The enzyme fatty acyl-CoA 
Table $\mathbf{I}$. Changes in the concentrations of plasma non-esterified fatty acids and blood ketone bodies $(\mu \mathrm{mol} / \mathrm{ml})$ in a number of species subjected to starvation

\begin{tabular}{|c|c|c|c|c|c|c|c|}
\hline & \multicolumn{2}{|c|}{ Fatty acids } & \multicolumn{2}{|c|}{ Acetoacetate } & \multicolumn{2}{|c|}{ 3-Hydroxybutyrate } & \multirow[b]{2}{*}{ References } \\
\hline & Fed & Starved & Fed & Starved & Fed & Starved & \\
\hline Dogfish & 0.15 & 0.14 & 0.07 & 0.42 & 0.03 & I. 86 & Zammit \& Newsholme, I 979 \\
\hline Bass & 0.90 & $I \cdot 50$ & 0.04 & 0.04 & $<0.01$ & $<0.01$ & Zammit \& Newsholme, I 979 \\
\hline Pigeon & - & - & 0.02 & 0.03 & 0.13 & 0.62 & Bailey \& Horne, 1972 \\
\hline Fowl & 0.32 & 0.99 & 0.06 & 0.10 & 0.37 & $1 \cdot 5^{\circ}$ & Davis \& Langslow, I 975 \\
\hline Cow & 0.35 & $2 \cdot 20$ & 0.04 & 0.09 & 0.37 & $1 \cdot 03$ & Baird et al. 1972 \\
\hline Rat & 0.10 & 0.57 & 0.10 & 0.80 & 0.08 & I. 79 & Berry et al. 1964 \\
\hline
\end{tabular}

synthase is located on the inner surface of the outer mitochondrial membrane (Nimmo, 1979) and in the microsomes (Norum et al. 1966; van Tol \& Hülsmann, 1970; De Jong \& Hülsmann, 1973). A fatty acyl-CoA synthase with preferential activity towards medium-chain fatty acids is present in the mitochondrial matrix (see, e.g., Groot et al. 1974, 1976; Bloch \& Vance, 1977). However, the function of this intramitochondrial synthase activity is not immediately apparent since, in the adult mammal, the liver receives primarily long-chain fatty acids from the circulation. Similarly, the significance of the dual distribution of the long-chain acyl-CoA synthase is difficult to ascertain, although it is possible that the mitochondrial enzyme gives rise to a distinct pool of long-chain acyl-CoA within the intermembrane space of the mitochondria (see below).

The partition of, and competition for, long-chain acyl-CoA between acylglyceride formation and acylcarnitine formation would be expected teleologically to be of major regulatory importance. That this is the case can be deduced from several observations that the relative proportions of fatty acids which are metabolized to glycerolipids or to oxidation products vary inversely in liver preparations obtained from animals in different physiological states (see, e.g., Mayes \& Felts, 1967; McGarry \& Foster, 1972; Laker \& Mayes, 1982). The deduction that this inverse relationship is due to competition between the first enzymes of the two pathways for their common substrate stems from observations that (i) if acylcarnitine formation is specifically inhibited the rate of glycerolipid synthesis is increased and reaches maximal rates even in livers from rats in ketogenic states (McGarry et al. 1973; Ide \& Ontko, 1981), and (ii) that it is only the proportions of fatty acids metabolized to glycerolipids or oxidation products which are altered in different physiological states, whereas the absolute rates of both processes are elevated with increased fatty acid supply (see, e.g., Laker $8 x$ Mayes, 1982). The regulation of the partition of long-chain acyl-CoA has been investigated intensively. Current theories about the mechanisms involved are centred around the study of the properties, changes in activities and of concentrations of substrates of the first enzymes of either pathway, namely carnitine acyltransferase I (CAT I) for oxidation, and glycerol-3-phosphate acyltransferase (GPAT) and dihydroxyacetone-phosphate acyltransferase 
(DHAPAT) for acylglyceride formation. For the purposes of the present review only the regulation of CAT I and GPAT will be considered since there is considerable controversy about the importance of DHAPAT activity in acylglyceride formation (see Cronholm et al. 1982, for a comprehensive discussion).

Changes in the concentrations of the non-common substrates of CAT I and GPAT (i.e. glycerol-3-phosphate and L-carnitine respectively) have been suggested by various workers to be involved in the modulation of long-chain acyl-CoA metabolism (McGarry et al. 1975; Robles-Valdez et al. 1976; Lund et al. 1980; Declercq et al. 1982). However, the concentrations of these metabolites in freezeclamped liver samples obtained from anaesthetized animals in different experimental and/or physiological states (Williamson et al. 1969; Zammit, 1981 $a$ ) have shown no consistent correlation with the rates of fatty acid oxidation or esterification. However, reports by different workers on changes in L-carnitine concentrations in liver of rats after short-term starvation vary considerably (Pearson \& Tubbs, 1967; Robles-Valdez et al. 1976; Brass \& Hoppel, 1978; Zammit, I98I $a$ ).

Changes in the rates of acylcarnitine and acylglyceride formation by isolated rat liver mitochondria, in the direction expected from the changes in the fate of fatty acids in the liver, have been described by several authors (Aas \& Daae, 1971; van Tol, 1974; Bremer et al. 1976). In addition, the activities of mitochondrial and microsomal GPAT are susceptible to short-term changes after treatment of rat liver preparations in vitro with hormones (Bates et al. 1977; Soler-Argilaga et al. 1978; Saggerson, 1979; Saggerson et al. 1981) and GPAT activity in hepatic microsomes and mitochondria can be inhibited in vitro by incubation with ATP$\mathrm{Mg}$ (Nimmo, 1980). These observations suggest that GPAT activity may be regulated through covalent modification of the enzyme (Nimmo, 1980). Although changes in activity which are specific to mitochondrial GPAT have been reported (Bates \& Saggerson, 1979; Zammit, 1981 $a$ ) for livers of starved rats, the function of the mitochondrial location of GPAT is uncertain, especially in view of the fact that mitochondria cannot metabolize the acylglyceride formed beyond the formation of phosphatidate (see Saggerson \& Bates, I98I, for discussion). Some evidence for the involvement of changes in the activity of GPAT in determining the rate at which long-chain acyl-CoA esters are esterified to glycerolipids has been obtained from the correlation between the total hepatic GPAT activity and the proportion of fatty acids used for glycerolipid formation in livers of fed or starved rats in various reproductive states (Zammit, $198 \mathrm{r} a$ ).

It is now recognized that although there may be an increase in the maximal activity of CAT I. in liver mitochondria from animals in ketogenic states, a far more powerful mechanism of regulation of the enzyme is the inhibitory effect of micromolar concentrations of malonyl-CoA on CAT I, competitively with respect to long-chain acyl-CoA (McGarry et al. 1977, 1978). Malonyl-CoA is the product of the first reaction committed to fatty acid synthesis (catalyzed by acetyl-CoA carboxylase). The activity of acetyl-CoA carboxylase (which is potentially rate- 
limiting for fatty acid synthesis) and the concentration of malonyl-CoA in the liver are both depressed in the liver of animals in ketogenic states (Guynn et al. 1972; Cook et al. 1977; Lynen, 1979; Zammit \& Corstorphine, 1982). Hence, in conditions in which fatty acid synthesis is depressed, the activity of CAT I becomes de-inhibited due to a decrease in cytosolic concentration of malonyl-CoA. Since the rate of fatty acid synthesis in liver is proportional to the concentration of malonyl-CoA (Guynn et al. 1972), the inhibition of CAT I by malonyl-CoA effectively ensures the integrated and reciprocal regulation of the flux through the two pathways such that fatty acid synthesis and oxidation do not occur to significant extents simultaneously in the liver. The fact that malonyl-CoA inhibition is competitive with respect to acyl-CoA (i.e. can be overcome at high concentrations of long-chain acyl-CoA) may also be important in integration of the flux through the two pathways. Thus, a rise in hepatic long-chain acyl-CoA concentration due to increased delivery of fatty acids to the liver would result in (i) a direct de-inhibition of CAT I activity through increased competition with malonyl-CoA, and (ii) a malonyl-CoA concentration lowering effect due to inhibition of acetyl-CoA carboxylase activity by long-chain acyl-CoA (see Goodridge, 1972). If a distinct pool of long-chain acyl-CoA esters does exist in the intermembrane space, the regulation of its size may be critical due to effect (i) above. Furthermore, a rationale for the location of a mitochondrial GPAT (with a high affinity for long-chain acyl-CoA (see Borrebaek et al. 1976; Bates \& Saggerson, 1979) on the inner aspect of the outer mitochondrial membrane (Nimmo, 1979; Carroll et al. 1982) may reside in the maintenance of a low concentration of long-chain acyl-CoA in the vicinity of CAT I under conditions in which acyl-carnitine formation is required to be minimal.

The mechanism of malonyl-CoA inhibition of CAT I has not been elucidated. For example, it is not known whether malonyl-CoA is an allosteric effector. However, there have been some recent discoveries which suggest that the mechanism of the effect of malonyl-CoA is more intricate than had hitherto been thought. The sensitivity to malonyl-CoA inhibition of the rate of ketogenesis from long-chain fatty acids in isolated liver mitochondria was higher in mitochondria from fed rats than in those from starved animals (Cook et al. 1980; Ontko \& Johns, I980). This effect was later shown to be reflected in the altered sensitivity of CAT I activity to malonyl-CoA in isolated mitochondria (McGarry \& Foster, I98I; Saggerson \& Carpenter, 198I ; Robinson \& Zammit, 1982). Moreover, experiments using fed or starved, unmated, pregnant or lactating rats (in which the range of hepatic malonyl-CoA concentrations is much larger than in fed and starved unmated animals (Zammit, I98Ia)) have demonstrated a quantitative relationship between the sensitivity of CAT I to malonyl-CoA inhibition in isolated liver mitochondria and the hepatic malonyl-CoA concentration in vivo (Figs. $2 a$ and $2 b$ ) (Robinson \& Zammit, 1982). These results suggest that CAT I in isolated mitochondria retains information about the concentration of malonyl-CoA in the liver from which they were isolated. The mechanism whereby this information is acquired and retained by the enzyme is not known, but two possibilities are 


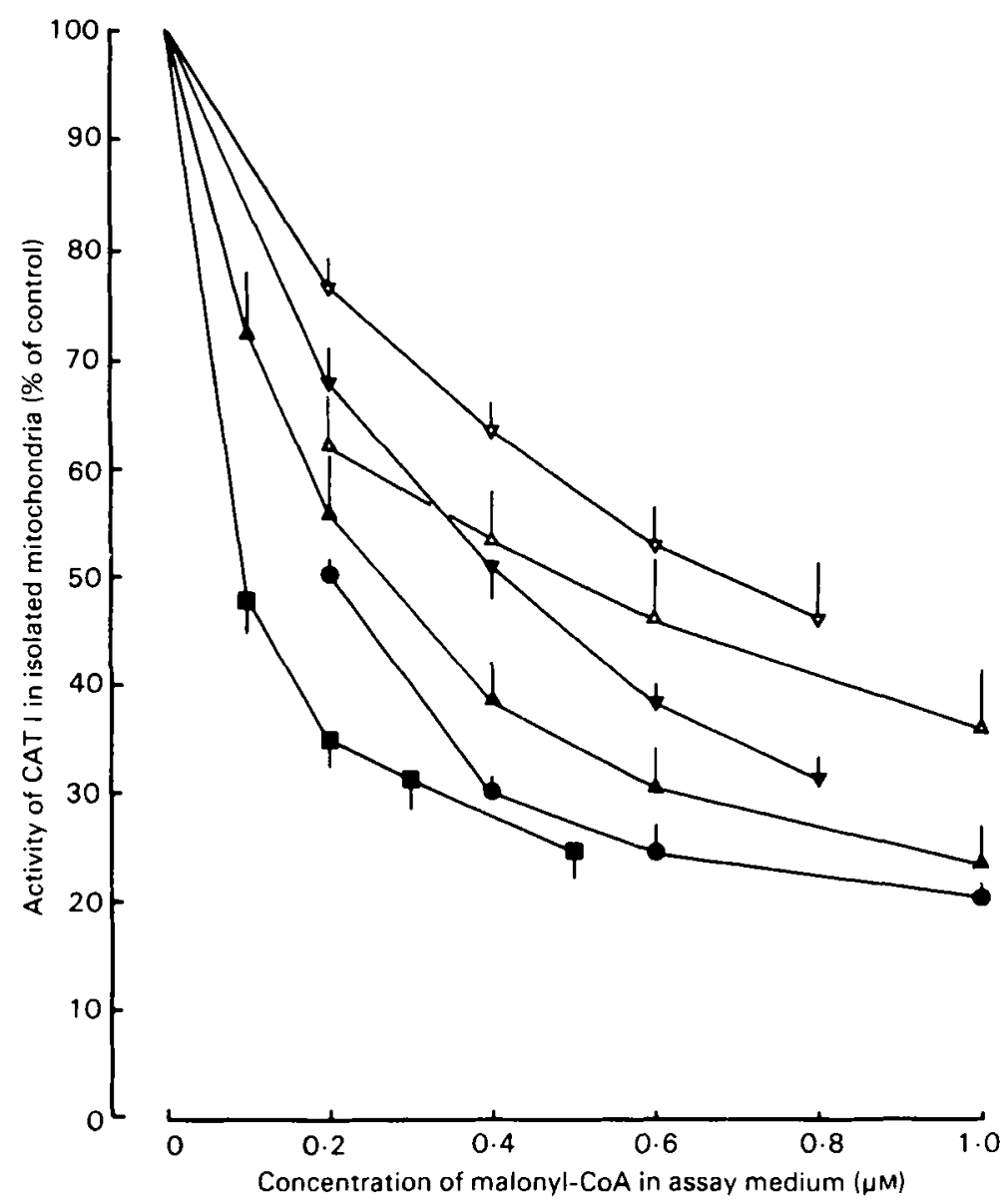

Fig. 2a. Effect of increasing malonyl-CoA concentrations on the activity of carnitine acyltransferase I (CAT I) in mitochondria isolated from livers of female rats in various physiological states: virgin $(\nabla, \nabla)$; late pregnant $(O, \Theta)$; peak-lactating $(\Lambda, \Delta)$; late lactating ( $\boldsymbol{\square})$ (from Robinson \& Zammit, 1982). Open symbols signify 24 h-starved animals.

(i) that CAT I is covalently modified by the same or a closely-related mechanism to that which is involved in the regulation of malonyl-CoA concentration (e.g. phosphorylation-dephosphorylation of acetyl-CoA carboxylase) and (ii) that CAT I tightly binds an effector molecule which continues to be bound during preparation of the mitochondria (in a manner analogous to the recently discovered binding of fructose 2,6-bisphosphate by phosphofructokinase in liver homogenates (Claus et al. 1980; Pilkis et al. 198I)). In the latter case the effector molecule could be either malonyl-CoA itself or another intermediate the concentration of which is regulated in parallel with that of malonyl-CoA (Robinson \& Zammit, r 982).

In order to obtain information about whether malonyl-CoA could be such an effector molecule, experiments were conducted using mitochondria isolated from livers of starved pregnant rats (in which CAT I is normally very insensitive to malonyl-CoA inhibition). The mitochondria were incubated either in the absence 


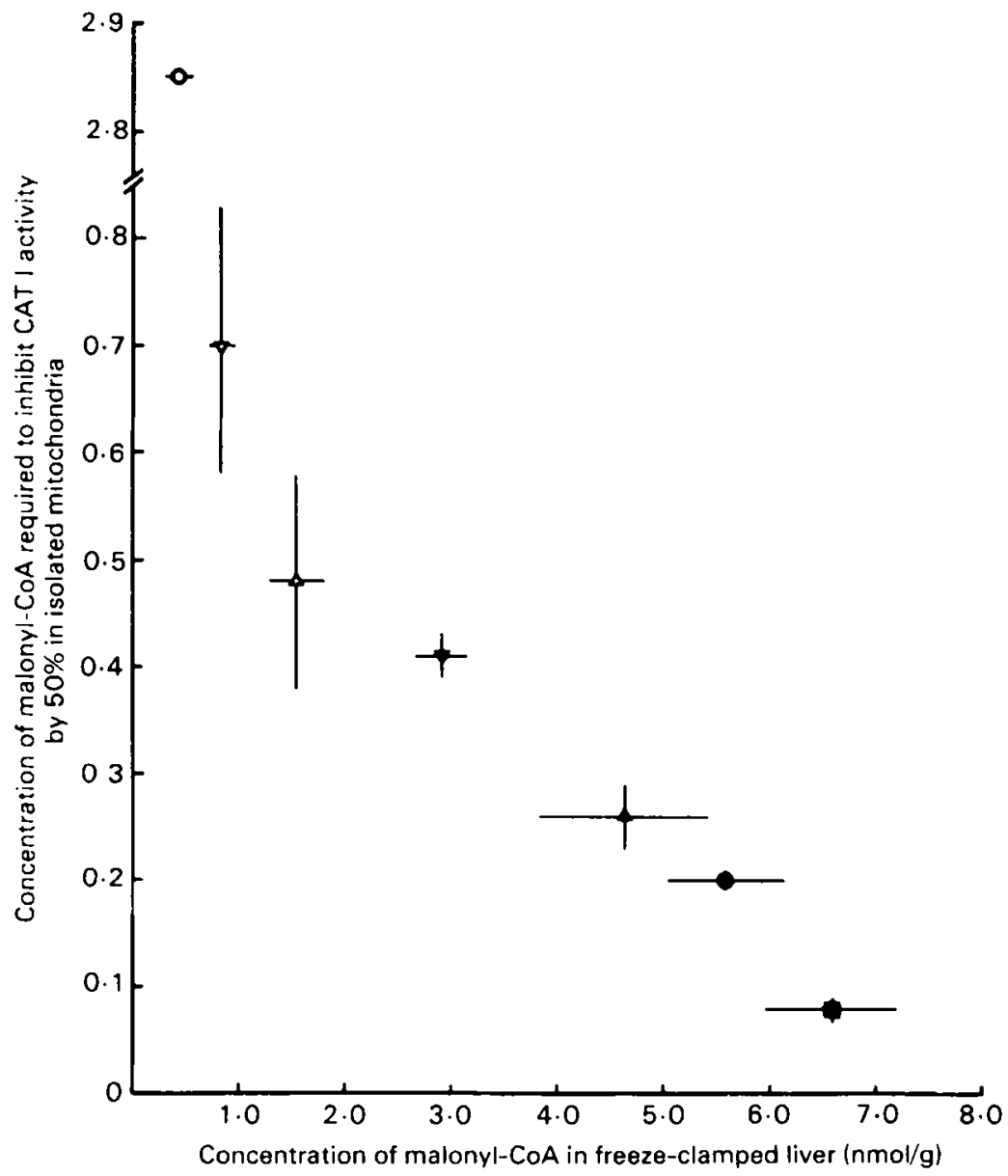

Fig. 2b. Relationship between the concentration of malonyl-CoA required to inhibit carnitine acyltransferase I (CAT I) activity by $50 \%$ in vitro (assayed in the presence of $40 \mu \mathrm{M}$-palmitoyl$\mathrm{CoA}$ and $10 \mathrm{mg}$ albumin/ml) and the concentration of malonyl-CoA in freeze-clamped liver (from Zammit, ı $981 a$; Robinson 8 Zammit, 1982). Notations are as given for Fig. $2 a$.

or in the presence of various concentrations of malonyl-CoA and were subsequently separated from the medium by sedimentation and filtration through an oil layer. The sensitivity of CAT I to malonyl-CoA inhibition was then investigated as previously described (Robinson \& Zammit, 1982). Following incubation of the mitochondria with malonyl-CoA, CAT I activity was significantly more sensitive to inhibition. That this increased sensitivity was not due to contamination of the mitochondrial preparation by malonyl-CoA was apparent from the fact that the activity of CAT I in the absence of malonyl-CoA was virtually identical in mitochondria which had been incubated either in the absence or in the presence of malonyl-CoA (Fig. 3).

Irrespective of the exact mechanism through which $\mathrm{CAT} I$ obtains and retains information about the malonyl-CoA concentration in vivo, it is evident that the 
self-reinforcing effects of the changes in malonyl-CoA concentration in the liver (i.e. increased concentrations resulting in increased sensitivity) could result in a potent amplification mechanism for the regulation of the rate of acylcarnitine synthesis by very small changes in hepatic malonyl-CoA concentration (Robinson \& Zammit, 1982).

Acylcarnitines are formed in the intermembrane space of the mitochondria and they have to be transported across the inner mitochondrial membrane for metabolism of the acyl moiety to proceed further. This transfer is mediated by a specific carrier-the carnitine-acylcarnitine translocase (Pande, 1975; Ramsay \& Tubbs, 1974, 1975; Pande \& Parvin, 1976, 1980b). This carrier mediates acylcarnitine-acylcarnitine and acylcarnitine-carnitine exchange although longer-chain acylcamitines are much better substrates than L-camitine itself (Ramsay, 1978). The initial rate of carnitine exchange has been shown to be increased in

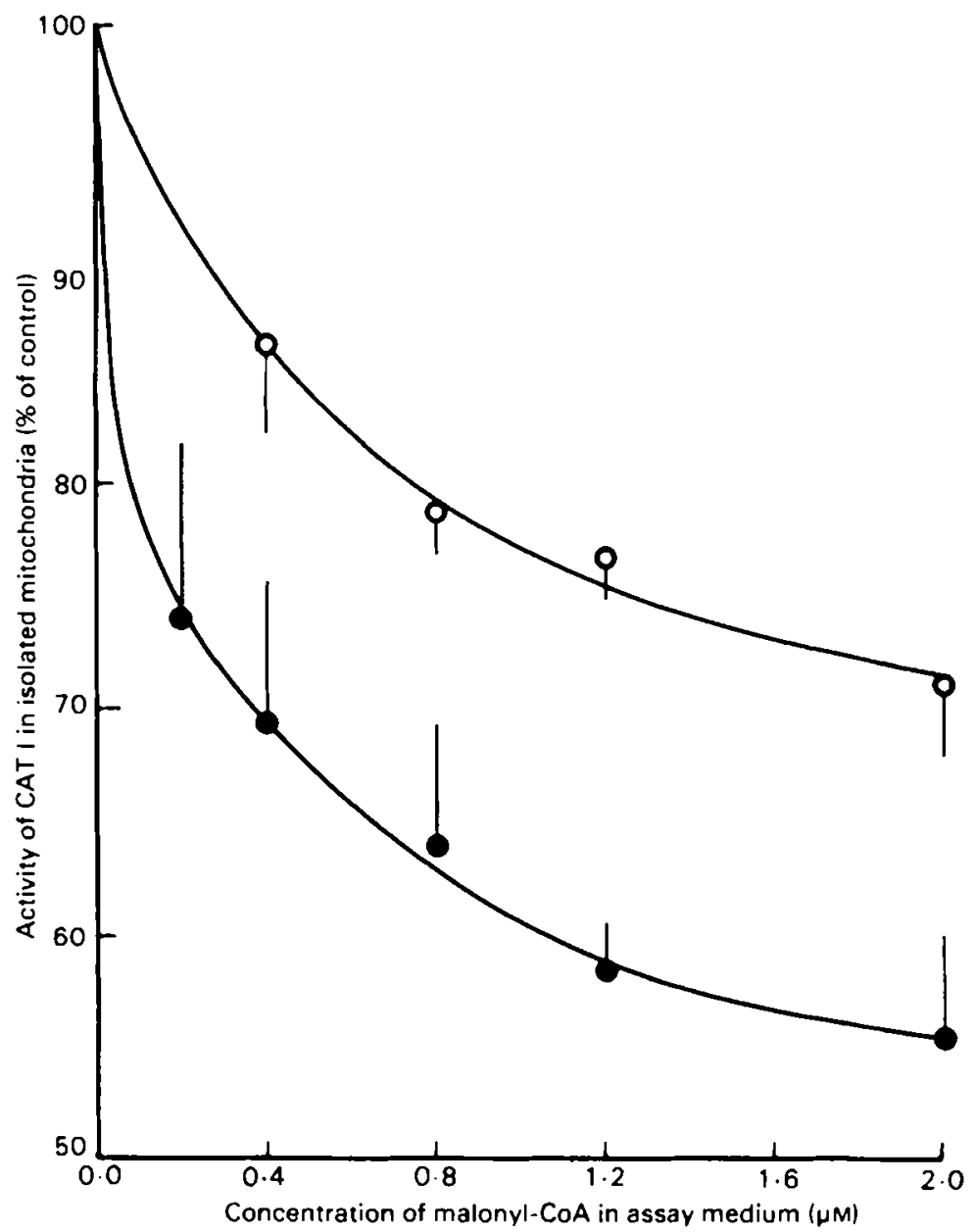

Fig. 3. Effect of incubation of mitochondria isolated from livers of starved, late-pregnant rats in the absence $(O)$ or presence $(O)$ of $65 \mu \mathrm{M}$-malonyl-CoA on the subsequent sensitivity of carnitine acyltransferase I (CAT I) activity to malonyl-CoA inhibition (see text for details). Values are means with their standard errors for three separate mitochondrial preparations. 
mitochondria isolated from livers of rats in ketogenic states (Parvin \& Pande, 1979) and this increase in the activity of the translocase was apparently due to an increased pool size of exchangeable carnitine (as unesterified L-carnitine and acylcarnitines) in these mitochondria (Parvin \& Pande, 1979; Pande \& Parvin, 1980a). Support for the possible involvement of an increased rate of acylcarnitine transport in the increased rate of hepatic oxidation of fatty acids in starvation, was obtained from experiments in which the rates of octanoylcarnitine-stimulated or octanoate-stimulated oxygen consumption of mitochondria, isolated from livers of fed or starved rats, were measured (Zammit, $1981 a$ ). Starvation resulted in a significant increase in octanoylcarnitine oxidation whereas it had no effect on octanoate-stimulated oxygen consumption: similar results were obtained by McGarry \& Foster (1974) using isolated perfused livers. Although these results would tend to exclude the possibility of $\beta$-oxidation as being a regulatory site for the increased rate of fatty acid oxidation, they do not distinguish between increased rates of acylcarnitine transport across the inner membrane and increased activity of CAT II (which resides on the inner face of the inner membrane and catalyzes the reverse reaction to that catalyzed by CAT I).

Intramitochondrial acetyl-CoA can be used either for the synthesis of citrate (by combining with oxaloacetate) or for the synthesis of acetoacetyl-CoA. The two enzymes involved are citrate synthase and acetyl-CoA acetyltransferase respectively. The formation of acetoacetyl-CoA is the first step committed to ketogenesis in liver mitochondria and the fact that acetyl-CoA acetyltransferase competes with citrate synthase for acetyl-CoA suggests that changes in the rate of citrate synthesis would inversely affect acetoacetyl-CoA synthesis. The rate of citrate synthesis is determined by at least two factors: (i) the relative affinities of citrate synthase and acetyl-CoA acetyltransferase for their common substrate, and (ii) the availability of free oxaloacetate in the mitochondria. Experiments with isolated rat liver mitochondria indicate that formation of citrate competes successfully for acetyl-CoA, i.e. that citrate synthase has a greater affinity for acetyl-CoA than acetyl-CoA acetyltransferase in situ (Lopes-Cardozo et al. 1975). Thus, progressively higher rates of fatty acid oxidation (i.e. acetyl-CoA formation) are accompanied by the rapid saturation of citrate formation followed by a steady increase in the rate of ketone body formation. If these results can be extrapolated to the situation in vivo they would predict that the maintenance of flux through the tricarboxylic acid cycle would have a higher metabolic priority than ketone body formation at any one level of oxaloacetate availability inside the mitochondria. Experiments in which the fates of ${ }^{14} \mathrm{C}$-labelled long-chain fatty acids were studied in perfused, isolated livers from fed or starved rats, have shown that the rates of ${ }^{14} \mathrm{CO}_{2}$ production were unaltered even though the rates of ketogenesis were markedly different in the two types of liver (McGarry \& Foster, 1974), suggesting that the energy-forming function of the tricarboxylic acid cycle was maintained. Although these experiments emphasize the importance of a more or less constant flux through the tricarboxylic acid cycle in the liver (see Mayes \& Laker, 198r), the overall rate of citrate synthesis must have decreased in livers of starved animals 
because de novo fatty acid synthesis, which in the rat depends on export of citrate from the mitochondria, was greatly diminished in these livers (McGarry \& Foster, 1974). Limitation of utilization of citrate for export from the mitochondria may be achieved through inhibition of citrate efflux across the inner membrane via competition by increased intramitochondrial concentrations of phosphoenolpyruvate in ketogenic-gluconeogenic states (Zammit, $198 \mathrm{I} b$ ) and by decreased availability of oxaloacetate for citrate synthesis. The antiketogenic action of alanine when infused into starved or diabetic rats has been ascribed to the increased availability of intramitochondrial oxaloacetate (Nosadini et al. 1980, I98I) and the free oxaloacetate concentration in mitochondria of isolated hepatocytes has been found to be inversely related to the rate of ketone body formation when the free oxaloacetate concentration is in the same range as the $K_{\mathrm{m}}$ of citrate synthase for oxaloacetate (Siess et al. 1982). The availability of oxaloacetate for citrate synthesis will, in turn, depend on (i) the rate of formation from pyruvate and its precursors (e.g. alanine, lactate) (ii) the equilibrium between oxaloacetate and malate, which will reflect the intramitochondrial [NAD]:[NADH] value, and (iii) the utilization of oxaloacetate for the formation of phosphoenolpyruvate (PEP) - either through the formation of malate or aspartate for efflux into the cytosol (in livers of species in which PEP carboxykinase is primarily cytosolic, e.g. the rat) or for formation of PEP directly inside the mitochondria (e.g. in the rabbit, ruminants and birds). The combination of these factors will result in more or less oxaloacetate being available for citrate synthesis. If citrate synthase is not saturated with oxaloacetate, increased availability of the latter results in decreased utilization of acetyl-CoA for acetoacetyl-CoA synthesis.

Although regulation of the partition of acetyl-CoA could be visualized in terms of changes in substrate concentration, both citrate synthase and acetyl-CoA acetyltransferase could be subject to metabolic control by effectors. However, the mode of regulation of these two enzymes in the liver is not easily ascertained. Several factors may affect citrate synthase, including the intramitochondrial ATP (Garland, 1968) and citrate (Bremer \& Davis, 1974) concentrations, but their importance remains ill-defined (Lopes-Cardozo \& van den Bergh, 1972; Siess et al. 1982). The regulatory properties of acetyl-CoA acetyltransferase have attracted appreciable interest in recent years. Liver mitochondria contain two forms of the enzyme which are interconvertible by binding (or loss) of coenzyme-A (Huth, I98I). Since the two forms have different kinetic characteristics (Huth et al. 1973) this interconversion may result in a high sensitivity of the activity of the enzyme to changes in the ratio of [acetyl-CoA]:[CoA]. Huth et al. (1975) have pointed out that the activity of the enzyme in the direction of acetoacetyl-CoA synthesis is very much lower than in the thiolytic direction, and from isotopic distribution studies they concluded that the activity of the enzyme may be rate-limiting (Huth et al. 1973). One factor which could limit acetyl-CoA acetyltransferase activity in vivo is inhibition by the product of the reaction, acetoacetyl-CoA (Huth et al. 1975). Acetoacetyl-CoA also inhibits 3-hydroxy-3-methyl glutaryl (HMG)-CoA synthase (Reed et al. 1975) - the next enzyme in the ketogenic pathway. These effects, 
coupled with the very low $K_{\mathrm{m}}$ of HMG-CoA synthase for acetoacetyl-CoA (Page \& Tubbs, 1978) suggest that the rate of formation of intramitochondrial HMG-CoA could be very sensitive to changes in the concentration of acetoacetyl-CoA and that the concentration of this metabolite needs to be regulated finely.

Huth et al. (1978) have suggested that intramitochondrial acetoacetyl-CoA concentrations could be determined by the state of the equilibrium catalyzed by 3-hydroxybutyryl-CoA dehydrogenase, which in turn will depend on the mitochondrial $\left[\mathrm{NAD}^{+}\right]:[\mathrm{NADH}]$ value. The increased rate of formation of NADH during fatty acid oxidation would result in lower concentrations of acetoacetyl$\mathrm{CoA}$. Another possible mechanism would involve the enzyme 3-oxoacid-CoA transferase (Zammit, $198 \mathrm{I} b$ ) which is as active as HMG-CoA synthase in the liver of mammals and more active in some other vertebrate species (Zammit et al. 1979). Interestingly, the mitochondrial $\left[\mathrm{NAD}^{+}\right]:[\mathrm{NADH}]$ value would also be involved in the latter mechanism which implies that the intramitochondrial concentrations of acetoacetate and succinyl-CoA should be maintained at a low level during high rates of ketogenesis. There is some experimental evidence to support this hypothesis. Thus, the mitochondrial succinyl-CoA level decreases by half in cells treated with glucagon (Siess et al. 1980), and Demaugre et al. (1978) found a three-fold lower [acetoacetate]:[3-hydroxybutyrate] value in the mitochondria than the cytosol in cells incubated with pyruvate. The concentration of acetoacetate would be expected to be maintained at a low level during increased fatty acid oxidation through increased formation of 3 -hydroxybutyrate (which is $\mathrm{NADH}$-linked) and increased efflux of acetoacetate from mitochondria in exchange for pyruvate (Halestrap, I $978 a, b$ ). Maintenance of low concentrations of succinyl$\mathrm{CoA}$ and acetoacetate would help to attain de-inhibition of acetyl-CoA acetyltransferase and thus allow ketogenesis to proceed. These considerations raise the possibility that feedback control of ketogenesis by ketone bodies occurs not solely in extrahepatic tissues (Williamson \& Whitelaw, 1978) but also at the site of ketogenesis, namely the liver mitochondria.

Table 2. Maximum activities ( $\mu \mathrm{mol} / \mathrm{min}$ per $g$ wet wt) of 3-hydroxy-3methyl glutaryl (HMG)-CoA synthase (EC 4.1.3.5), 3-oxoacid-CoA transferase (EC 2.8.3.5) and D-3-hydroxybutyrate dehydrogenase (EC I.1.1.30) in liver of several vertebrate species*

\begin{tabular}{|c|c|c|c|c|}
\hline \multirow[b]{2}{*}{ Animal } & \multicolumn{4}{|c|}{ Enzyme activities } \\
\hline & $\begin{array}{l}\text { Temperature of } \\
\text { measurement }\left({ }^{\circ}\right)\end{array}$ & $\begin{array}{c}\text { HMG-CoA } \\
\text { synthase }\end{array}$ & $\begin{array}{l}\text { 3-Oxoacid-CoA } \\
\text { transferase }\end{array}$ & $\begin{array}{c}\text { 3-Hydroxybutyrate } \\
\text { dehydrogenase }\end{array}$ \\
\hline Mackerel & 10 & 0.14 & 40 & $<0.01$ \\
\hline Bass & 10 & 0.25 & $9 \cdot 2$ & $<0.01$ \\
\hline Dogfish & Io & 0.41 & 0.8 & 0.16 \\
\hline Pigeon & 25 & $\mathrm{I} \cdot 7$ & $I \cdot 5$ & 0.15 \\
\hline Chicken & 25 & I. 7 & $I \cdot 0$ & 0.22 \\
\hline Rat & 25 & I. 8 & 0.4 & $12 \cdot 4$ \\
\hline
\end{tabular}


The regulatory mechanisms discussed above were either deduced from experiments on, or postulated for, the liver of the rat and, although aspects of ketogenesis have been studied in other species, the regulation of the pathway remains largely unexplored in animals other than the rat. However, the limited number of comparative studies which have been carried out have provided some valuable ideas. Thus, the suggestion that 3 -oxoacid-CoA transferase activity may be involved in the regulation of intramitochondrial acetoacetyl-CoA concentrations (Zammit et al. 1979; Zammit, 198 1 ) emerged as a result of the observation that the enzyme is present in liver of all vertebrate species studied, and that in the teleost fish the activity is extremely high (Zammit et al. 1979). Further investigations established that these animals could not form 3-hydroxybutyrate and could not use ketone bodies as an alternative substrate to glucose during starvation (Table 2; Zammit \& Newsholme, 1979). Other interesting possibilities arise from the comparative results already available. For example, in view of the apparent involvement of the intramitochondrial $\left[\mathrm{NAD}^{+}\right]:[\mathrm{NADH}]$ value in the regulation of ketogenesis, it would be intriguing to explore the significance of the extramitochondrial location of D-3-hydroxybutyrate dehydrogenase in liver of ruminants (Watson \& Lindsay, 1972); an investigation of the reciprocal regulation of fatty acid synthesis and oxidation by changes in hepatic malonyl-CoA in liver of ruminants would be of interest because of the low rate of hepatic fatty acid synthesis in these animals; and variations in integration of the rates of gluconeogenesis and ketogenesis may exist among species because of different patterns of distribution of PEP carboxykinase between the cytosol and the mitochondria. These and other questions still require to be answered and, when they are, they will yield not only interesting information about different biochemical strategies adopted by animals with different metabolic requirements but also about the basic mechanisms involved in the regulation of fatty acid oxidation and ketogenesis.

\section{REFERENCES}

Aas, M. \& Daae, N. W. (1971). Biochim. biophys. Acta 239, 208.

Bailey, E. \& Horne, J. A. (1972). Comp. Biochem. Physiol. 42B, 659.

Baird, G. D., Heitzman, R. J. \& Hibbit, K. G. (1972). Biochem. F. 128, 1 31 1.

Bates, E. J. \& Saggerson, E. D. (1979). Biochem. F. 182, 751.

Bates, E. J., Topping, D. L., Sooranna, S. R., Saggerson, E. D. \& Mayes, P. A. (1977). FEBS Lett. 84, 225 .

Berry, M. N., Williamson, D. H. \& Wilson, M. B. (1964). Biochem. F. 94, i 7 C.

Bloch, K. \& Vance, D. (1977). Ann. Rev. Biochem. 46, 263.

Borrebaek, B., Christiansen, R., Christophersen, B. O. \& Bremer, J. (1976). Circulation Res. $3^{8}$ (Suppl. I) I6.

Brass, E. P. \& Hoppel, C. L. (1978). 7. biol. Chem. 253, 2688.

Bremer, J., Bjerve, K. S., Borrebaek, B. \& Christiansen, R. (1976). Mol. Cell Biochem. I2, I 3.

Bremer, J. \& Davis, E. J. (1974). Biochim. biophys. Acta 370, 564.

Carroll, M. A., Morris, P. E., Grosjean, C. D., Anzalone, T. \& Haldar, D. (1982). Arch. Biochem. Biophys. 214, 17.

Claus, T. H., Schlumpf, J. R., El-Maghrabi, M. R., Pilkis, J. \& Pilkis, S. J. (1980). Proc. Nat. Acad. Sci., USA 77, 6501. 
Cook, G. A., Nielsen, R. C., Hawkins, R. A., Mehlman, M. A., Lakshmanan, M. R. \& Veech, R. L. (1977). F. biol. Chem. 252, 442 I.

Cook, G. A., Otto, D. A. \& Comell, N. W. (1980). Biochem. F. 192, 955.

Cronholm, T., Curstedt, T. \& Sjövall, J. (1982). In Metabolic Compartmentation, p. 33 I [H. Sies, editor]. London and New York: Academic Press.

Davis, T. F., \& Langslow, D. R. (1975). Comp. Biochem. Physiol. 52A, 645.

Declercq, P. E., Debeer, L. J. \& Mannaerts, G. P. (1982). Biochem. J. 204, 247.

De Jong, J. W. \& Hülsmann, W. C. (1973). Biochim. biophys. Acta 197, 127.

Demaugre, F., Leroux, J.-P. \& Cartier, P. (1978). Biochem. F. 172, 91.

Fine, M. B. \& Williams, R. H. (1960). Am. F. Physiol. 199, 403.

Garland, P. B. (1968). In Metabolic Roles of Citrate, p. 4 I [T. W. Goodwin, editor]. New York: Academic Press.

Goodridge, A. G. (1972). F. biol. Chem. $247,6949$.

Goresky, G. A., Daly, D. S., Mishkin, S. \& Arias, I. M. (1978). Am. F. Physiol. 234, E$_{542 .}$

Groot, P. H. E., Scholte, H. R. \& Hülsmann, W. C. (1976). Adv. Lipid Res. 14, 75.

Groot, P. H. E., van Loon, C. M. I. \& Hülsmann, W. C. (1974). Biochim. biophys. Acta 337, I.

Guynn, R. W., Veloso, D. \& Veech, R. L. (1972). F. biol. Chem. 247, 7325 .

Hales, C. N., Luzio, J. P. \& Siddle, K. (I 978). Biochem. Soc. Symp. 43, 97.

Halestrap, A. P. (1978a). Biochem. F. 172, 377 .

Halestrap, A. P. (1978b). Biochem. $\mathcal{7}$ 1 172, $3^{89}$.

Huth, W. (1981). Eur. F. Biochem. 120, 357.

Huth, W., Dierich, C., Deynhausen, V. \& Seubert, W. (1973). Hoppe-Seyler's Z. Physiol. Chem. 354,635 .

Huth, W., Jonas, R., Wunderlich, I. \& Seubert, W. (1975). Eur. Y. Biochem. 59, 475.

Huth, W. \& Menke, R. (1980). Hoppe-Seyler's Z. Physiol. Chem. 361, 607.

Huth, W., Stermann, R., Holze, G. \& Seubert, W. (1978). In Biochemical and Clinical Aspects of Ketone Body Metabolism, p. I I [H. D. Söling and C. D. Seufert, editors]. Stuttgart: George Thieme.

Ide, T. \& Ontko, J. A. (1981). F. biol. Chem. 256, 10247.

Laker, M. E. \& Mayes, P. A. (1982). Biochem. F. 206, 427.

Lopes-Cardozo, M., Mulder, I., Van Vugt, F., Hermans, P. G. \& van den Bergh, S. G. (1975). Mol. Cell Biochem. 9, 155.

Lopes-Cardozo, M. \& van den Bergh, S. G. (1972). Biochim. biophys. Acta 283, I.

Lund, H., Borrebaek, B. \& Bremer, J. (1980). Biochim. biophys. Acta 620, 364 .

Lynen, F. (1979). In Perspectives in Inherited Metabolic Diseases, vol. 3, p. I [B. Berra and S. DiDonato, editors]. Milan: Ermes.

McGarry, J. D. \& Foster, D. W. (1972). Metab. Clin. Exp. 21, 47 I.

McGarry, J. D. \& Foster, D. W. (1974). J. biol. Chem. 249, 7984.

McGarry, J. D. \& Foster, D. W. (1981). Biochem. F. 200, 217.

McGarry, J. D., Jürgen, M. M. \& Foster, D. W. (1973). J. biol. Chem. 248, 270.

McGarty, J. D., Mannaerts, G. P. \& Foster, D. W. (1977). F. clin. Invest. 60, 265.

McGarry, J. D., Mannaerts, G. P. \& Foster, D. W. (r978). Biochim. biophys. Acta 530, 305.

McGarry, J. D., Robles-Valdes, C. \& Foster, D. W. (1975). Proc. Nat. Acad. Sci., USA 72, 4385. Mayes, P. A. \& Felts, J. M. (1967). Nature, Lond. 2 15, 7 I6.

Mayes, P. A. \& Laker, M. E. (1981). Biochem. Soc. Trans. 9, 339.

Middleton, B. (1978). In Biochemical and Clinical Aspects of Ketone Body Metabolism, p. I [H. D. Söling and C. D. Seufert, editors]. Stuttgart: George Thieme.

Nestel, P. J. \& Steinberg, D. (1963). J. Lipid Res. 4, 46r.

Nimmo, H. G. (1979). FEBS Lett. 101, 262.

Nimmo, H. G. (1980). In Recently Discovered Systems of Enzyme Regulation by Reversible Phosphorylation, p. I 35 [P. Cohen, editor]. Amsterdam: Elsevier.

Norum, K. R., Farstad, M. \& Bremer, J. (1966). Biochem. biophys. Res. Commun. $22,797$.

Nosadini, R., Alberti, K. G. M. M., Johnston, D. G., Del Prato, S., Marescotti, C. \& Duner, E. (198I). Metab. clin. Exp. 30, 563.

Nosadini, R., Datta, M., Hodson, A. \& Alberti, K. G. M. M. (1980). Biochem. F. 190, 323.

Ontko, J. A. \& Johns, M. L. (1980). Biochem. J. r92, 959.

Owen, D. E., Felig, P., Morgan, A. P., Wahren, J. \& Cahill, G. F. (1969). F. clin. Invest. 48, 574. 
Page, M. A. \& Tubbs, P. K. (1978). Biochem. Y. 173, 925.

Pande, S. V. (1975). Proc. Nat. Acad. Sci., USA 72, 883.

Pande, S. V. \& Parvin, R. (1976). F. biol. Chem. 251, 6683.

Pande, S. V. \& Parvin, R. (1980a). J. biol. Chem. 255, 2994.

Pande, S. V. \& Parvin, R. (1980b). In Carnitine Biosynthesis, Metabolism \& Functions, p. I43

[R. A. Frenkel and J. D. McGarry, editors]. New York: Academic Press.

Parvin, R. \& Pande, S. V. (1 979). F. biol. Chem. 25, 5423.

Pearson, D. J. \& Tubbs, P. K. (1967). Biochem. F. 105, 953.

Pilkis, S. J., El-Maghrabi, M. R., Pilkis, J., Claus, T. H. \& Cumming, D. A. (1981). Y. biol. Chem. 256, 3171.

Ramsay, R. R. (1978). Biochem. Soc. Trans. 6, 72.

Ramsay, R. R. \& Tubbs, P. K. (1974). Biochem. Soc. Trans. 2, 1285.

Ramsay, R. R. \& Tubbs, P. K. (1975). FEBS Lett. 54, 21.

Reed, W. D., Clinkenbeard, K. D. \& Lane, M. D. (1975). J. biol. Chem. 250, 3 1 7 .

Robinson, I. N. \& Zammit, V. A. (1982). Biochem. F. 206, I 77.

Robles-Valdez, C., McGarry, J. D. \& Foster, D. W. (1976). F. biol. Chem. 25 I, 6007.

Saggerson, E. D. (1979). Trends Biochem. Sci. 4, 33.

Saggerson, E. D. \& Bates, E. (1981). In Short-term Regulation of Liver Metabolism, p. 247 [L. Hal and G. Van de Werve, editors]. Amsterdam: Elsevier.

Saggerson, E. D. \& Carpenter, C. A. (1981). FEBS Lett. 129, 225.

Saggerson, E. D., Snoswell, A. M., Trimble, R. P., Illman, R. J. \& Topping, D. L. (1981). Biochem. Int. 3, $44^{\mathrm{t}}$.

Siess, E. A., Fahimi, F. M. \& Wieland, O. H. (1980). Biochem. Biophys. Res. Commun. 95, 205.

Siess, E. A., Kientsch-Engel, R. I. \& Wieland, O. H. (1982). Eur. Y. Biochem. 12 I, 493.

Soler-Argilaga, C., Russel, R. L. \& Heimberg, M. (1978). Arch. Biochem. Biophys. 190, 367.

van Tol, A. (1974). Biochim. biophys. Acta 357, 14.

van Tol, A. \& Hülsmann, W. C. (1970). Biochim. biophys. Acta 223, 416.

Watson, H. R. \& Lindsay, D. B. (1972). Biochem. F. 128, 53.

Williamson, D. H., Veloso, D., Ellington, E. V. \& Krebs, H. A. (1969). Biochem. F. $114,575$.

Williamson, D. H. \& Whitelaw, E. (1978). Biochem. Soc. Symp. 43, 137.

Zammit, V. A. (1980). Biochem. Soc. Trans. 8, 543 .

Zammit, V. A. (1981a). Biochem. F. 198, 75.

Zammit, V. A. $(198 \mathrm{1} b)$. Trends Biochem. Sci. 6, 46 .

Zammit, V. A., Beis, A. \& Newsholme, E. A. (1979). FEBS Lett. 103, 2 I 2.

Zammit, V. A. \& Corstorphine, C. G. (1982). Biochem. F. 204, 757.

Zammit, V. A. \& Newsholme, E. A. (1979). Biochem. F. 184, 313. 